\title{
Research on Locomotive Energy-saving Control System
}

\author{
Xiangcai Wang \\ Jilin Railway Technology College, Jilin, Jilin, 132200
}

Keywords: energy-saving technology; control system; locomotive system

\begin{abstract}
The 11st "Five-Year Plan" of the country clearly puts forward the constraining indicators for energy conservation and consumption reduction in China. The total amount of energy consumed by railway transportation is very large, and the task of energy-saving in the railway industry is very arduous. The 11st "Five-Year Plan" for the development of railway science and technology promulgated by the Ministry of Railways clearly stipulates energy-saving and environmental protection technologies as the main areas of development, comprehensively carries out applied research and promotion of resource conservation, and improves the scientific and technological level of railway energy conservation.
\end{abstract}

\section{Introduction}

In recent years, the rapid development of computer control technology, communication technology and signal technology has provided technical support for the realization of a variety of operational control methods for locomotives. Through the study of train optimization maneuver technology, people have a deeper understanding of the train's operating methods, and the development of predictive control technology provides a better method for comparison and selection of the advantages and disadvantages of the operating plan. The locomotive energy-saving operation control system collects the running status of the train in real time, intelligently analyzes the data in connection with the line, predicts and determines a reasonable and feasible energy-saving operation plan, and assists the driver in completing the energy-saving operation on the specified line. China's railway science and technology workers have also carried out research on locomotive maneuver optimization methods in accordance with China's railway operations. Northern Jiaotong University uses a combination of off-line optimization calculation and on-line fuzzy control to develop a vehicle-mounted optimization guidance computer control system that is controlled by a single-chip microcomputer and has undergone a loading operation test. These efforts have played an active role in exploring and promoting the establishment of an energy-saving optimization manoeuvring method adapted to the locomotive operating environment in China. In recent years, the Bureau has done a lot of work in optimizing energy consumption structure, improving resource and energy use efficiency, reducing railway transportation costs, and improving the railway environment, and has achieved remarkable results. However, due to the opening of the new line, the speed of trains, the workload of passenger and freight trains are increasing, the change of type and traction of locomotives has changed, the workload of high-consumption transportation types increases, the impact of objective factors such as electricity price and rising oil prices, and the energy consumption of transportation The increase in cost has directly affected the management and management objectives of the Bureau of Roads [1]. To this end, we attach importance to energy conservation and consumption reduction, and focus on the positioning of the energy conservation work. That is, we must focus on the work of saving energy and reducing consumption of locomotives, which has become a topic of great concern.

\section{Train Energy-saving Optimization Maneuvering Strategy}

At the instant of the start of the train, the change in the acceleration of the train is a very short and short-lived process. Therefore, the acceleration change rate at the instant of train start is very large. If the rate of change of acceleration is too high, it will inevitably lead to serious impacts on 
the train and may even result in serious accidents such as injury or death to the passengers of the vehicle structure. Therefore, the starting acceleration and its rate of change must be limited in order to start safely and smoothly. The control of the train start-up phase follows the following principles of optimized operation:

In order to achieve a smooth starting purpose, the speed control handle needs to stay in the low level for a period of time to eliminate the clearance of the train coupler under less starting traction force. The specific truck can take 7 seconds and the bus can take 5 seconds. At the same time, attention should be paid to avoid that the starting traction is greater than the adhesion of the locomotive and that the wheel idling. In order to reduce the energy consumption to overcome the basic resistance, during the start of the train, it should be accelerated with the maximum acceleration under the premise of ensuring safety.

Since infinite acceleration does not exist in practice, the train should be accelerated at the maximum acceleration that can be achieved during the start and acceleration of the train. This reduces the basic resistance work during acceleration and reduces the time spent in the acceleration process. The kinetic energy will be inevitably lost when the brakes are stopped. Because the braking time savings means energy savings, the less time it takes to use the parking brake, the better. Therefore, braking at the maximum brake capacity of the train during the parking brake of the train is most beneficial to energy saving. To sum up, in order to achieve the energy-saving operation of the train, the non-uniformity of the train operating speed should be minimized, start with the maximum possible acceleration, try to ensure the smoothness of the speed during the run, and try to stop with the maximum possible braking capacity.

Consider the two modes of operation, constant speed operation and variable speed operation, both of which travel the same distance in the same time and have the same starting and ending speeds [2]. Therefore, only the basic resistance work of the two operation modes needs to be compared to obtain a uniform speed. The basic resistance work to overcome is minimal. When the train is running on the way, although uniform operation is most beneficial to energy conservation, it is difficult to achieve in the actual operation process, and can only be approached by continuous traction and laziness. As a result, for lines with small changes in level or slope, it is theoretically proven that there is an optimal control sequence for "maximum acceleration, uniform operation, coasting, maximum braking”, that is, when running on flat or less ramps, Reducing the non-uniformity of train running speed can reduce the basic resistance work during train operation. When the train starts, if the constraints of the same time, distance and end speed are taken into account, the train is accelerated to a certain speed with infinite acceleration, and then the distance constraint is completed at this speed, and finally the train to the terminal speed limit is accelerated with infinite acceleration. In this type of operation, the speed of unevenness is the smallest due to the infinite acceleration does not exist. In actual operation, the train is accelerated to the maximum speed with a maximum acceleration, and the train runs at a constant speed, accelerating the train to the end speed with the maximum acceleration. The most energy-saving maneuvering method is the condition, and this also applies to the acceleration running on the train. If you do not consider constraints such as time and distance, when starting the train, starting from the perspective of energy saving, the train should be accelerated with the maximum acceleration to reduce the train start time and create conditions for running at a lower speed in the future. In the actual starting, starting from the safety and stability of the train operation, starting with the maximum acceleration, it is easy to cause the dynamic impact of the vehicle. Considering that the proportion of the train startup during the whole operation is very small, the locomotive is in the initial stage of starting. The main control handle should be as low as possible. Slowly stretch the train. After the tail vehicle moves, gradually increase the main control handle. In the later stage of start-up, accelerate the train with the maximum traction, taking into account the smooth running and energy saving of the train.

\section{Prediction of Speed of Locomotive Energy-saving Operation}

When calculating the train resistance and braking force, it is necessary to know the traction weight of the train. The traction quality can be read from the box as a reference value for calculation. 
However, the traction quality actually read is input by the driver. The error is very large. It is necessary to calculate the traction quality of the train and feed back the calculation formula of braking force and resistance to make the calculation more accurate. Moreover, the traction quality largely determines the railway's ability to pass, transport and transport costs. There are many ways to calculate the traction mass, but many methods are used to specifically carry out the traction quality, for example, the calculation method of the traction weight when the acceleration is running at the highest speed on the straight pass, and the calculation of the traction weight when using the kinetic energy on the slope [3]. Methods, etc. These methods are almost impossible to use in the real-time traction quality calculation of trains. The method of traction quality calculated by Newton's second law algorithm is mainly considered here. In the speed prediction process, the speed of the train, distance traveled, and time of operation need to be calculated in different sections. The speed of the train, the time, and the distance are all determined by the resultant force acting on the train. Therefore, the relationship between the speed, distance, time, and resultant force of the train must be established. This is the application of the train equation of motion. If the resultant force acting on the train is a constant, the train's acceleration is a constant and the train moves at a constant speed. However, in reality, the resultant force acting on the train changes with the change of speed, so the train's movement is a complex non-uniform speed-changing movement, and the train's operation problem cannot be directly solved by the equation of motion. In order to calculate conveniently, it is usually to use segmented cumulative approximation method when solving the problem of train operation. This method divides train speed into intervals. If each interval is not significant, then in each speed interval, the resultant force acting on the train is treated as a constant for the nearsightedness, ie, the resultant force and acceleration corresponding to the average speed in each speed interval can be regarded as constant. In this way, the running distance and the running time in each speed interval can be calculated according to the relevant formula of the constant speed movement.

\section{Speed Prediction Analysis Based on Predictive Control}

The core idea of predictive control is rolling optimization. Regardless of the form of the algorithm, predictive control is based on the three major methodologies of accepted predictive control, namely model-based prediction, rolling optimization, and feedback correction. Model predictive control is a control algorithm based on the dynamic characteristics model describing the system. This model is called a predictive model. Its function is to predict the future output of the system based on the historical information of the controlled object and future input. The prediction process of locomotive energy-saving operation speed firstly establishes a model describing the dynamic characteristics of the system based on the predictive control algorithm, and this model is the speed measurement process analyzed in the section. This process is a speed prediction process, a speed prediction model. In the prediction model of locomotive running speed, according to the function of model predictive control, it is divided into the following sections of line profile information for locomotive speed prediction, including interval slope, interval length, interval bend radius and length, and interval start point end Elevation, tunnel conditions, ramp signals, etc. It also includes the locomotive parameters collected by the system itself, numerous analog quantities, switching quantities, and digital signals [4]. The controlled object is the speed of the locomotive. In a process or a cycle of speed prediction, the range is divided into three according to the different calculations. Interval, and set the end speed of the end zone according to the speed limit condition, and use this as the speed prediction of other points in the interval. This self-set end speed is the future input of the speed of the controlled object. The main purpose of the locomotive energy-saving operation control system is to calculate the speed according to the traction calculation model through known historical information, and to predict the speed with the locomotive optimization maneuvering strategy. The future output of the prediction system is the speed curve predicted after calculation of the interval.

Because of the difference in the level of the locomotive to operate the locomotive, the actual running speed of the locomotive after a certain distance may have a large error with the predicted 
speed. Therefore, the error should be corrected in time to predict the forward speed running curve at the current speed again. Although there may have been a large deviation between the actual speed and the predicted speed before, after the second forecast, the driver can drive at the current speed. That is, the system optimizes the speed prediction curve through a rolling method so that the locomotive can calculate the best energy-saving operation prediction speed in the current state at any position. This is also consistent with predictive control at each moment with a performance optimization index relative to that moment, which is the forecast speed. The relative form of the speed forecasting indicators at different times of the system is the same, but its absolute form, that is, the contained time zone and location zone are different. The system provides an energy-saving operation and control scheme at any time at any location. The system is a non-stop real-time calculation process. From the entire process of control, this optimization process is dynamic. The design of the system perfectly reflects the idea of predictive control rolling optimization.

In the system velocity prediction process, one cycle predicts the output of a prediction curve, and the actual velocity of the locomotive at different positions deviates from the predicted velocity. The feedback correction of the system is to feed back the actual velocity that deviates from the predicted velocity to the velocity prediction model to re-estimate. The speed curve makes the deviation of the actual speed from the prediction curve smaller and smaller. When the coincidence between the actual running curve of the locomotive and the predicted curve becomes larger, the energy saving effect of the locomotive will be more obvious. Each forecasting process in the speed forecasting model is dynamic and different, that is, each forecasting process is divided into different intervals according to the length of the interval. The number of intermediate intervals is generally different, and each internal interval must be calculated once [5]. The optimization of the speed prediction control is based not only on the model but also on the feedback information, thus constituting a closed-loop optimization.

\section{Conclusion}

This article describes the establishment of a prediction model for locomotive energy-saving operating speed. The model integrates the locomotive energy-saving maneuvering strategy, the train force traction calculation model, and the line profile information, and the fuzzy predictive control idea is well penetrated into the establishment of the model. The application of fuzzy control and predictive control in the speed prediction model solves the problem of combining human expert experience, knowledge, and computer real-time forecasting calculations. It is a major breakthrough.

\section{References}

[1] Li Yusheng, Hou Zhongsheng. Research on Train Energy Saving Control Based on Genetic Algorithm [J]. Journal of System Simulation. 2007 (02)

[2] He Yi, Xu Ling. Application of CF Card in Microcontroller System [J]. Test Technology of China. 2006 (02)

[3] Li Yusheng. Application of Genetic Algorithm in Train Energy Saving Optimization [J]. Shanxi Science and Technology. 2006 (02)

[4] Li Ping, Zhang Jian, Zhou Zhen, Yin Mingjuan. Development of intelligent optimization guidance device for locomotives [J]. Instrumentation Users. 2006 (01)

[5] Wu Yang, Luo Xia. A real-time adjustment strategy and its dynamic speed control model for late subway train [J]. China Railway Science. 2005 (06) 